\title{
Nucleophilic Addition of Potassium $O$-Ethyl Dithiocarbonate to Baylis-Hillman Adducts Using 9-BBN as Catalyst
}

\author{
Ilsa Hernández-Ibinarriaga, and Luis D. Miranda* \\ Instituto de Química, Universidad Nacional Autónoma de México, Circuito Exterior, Ciudad Universitaria, \\ México, D.F. 04510, México
}

Received May 11, 2009; accepted June 26, 2009

\begin{abstract}
An efficient diastereoselective Michael addition of the commercial potassium O-ethyl dithiocarbonate to Baylis-Hillman adducts in the presence of 9-BBN (9-borabicyclo[3.3.1]nonane) as a Lewis acid is reported. 9-BBN both protected the hydroxyl group and activated the carbonyl of the Michael acceptor.

Keywords: Xanthates; Baylis-Hillman adducts; 9-BBN as a Lewis acid.
\end{abstract}

\section{Introduction}

The densely functionalized Baylis-Hillman adducts have considerable preparative value in the synthesis of complex molecules [1].Most Michael addition processes on Baylis-Hillman adducts involve the loss of the hydroxyl group, an important functional group in the adduct structure. Except for a few documented cases [2], the loss of this group is typically prevented by incorporating an undesirable extra alcohol-protection/deprotection sequence into the synthetic plan [3].In recent years xanthates (dithiocarbonates)[4] have found widespread applications in organic syntheses of complex structures and in living radical polymerization chemistry, mainly exploiting the efficiency of these compounds in the production of alkyl free radicals [5].

\section{Results and discussion}

In recent efforts to synthesize the xanthate $\mathbf{3}$, we examined the nucleophilic addition of the commercial potassium O-ethyl dithiocarbonate to a Baylis-Hillman adduct without the loss of the hydroxyl group (Scheme 1). Specifically, we examined the reaction of the xanthate salt with the Baylis-Hillman adduct 1 in acetonitrile and acetic acid (as a proton source), stirring the reaction mixture at different temperatures. However, after $24 \mathrm{~h}$ of reaction, most of the starting hydroxyester 1 was recovered unchanged and a 1:1 diastereoisomeric mixture of the desired xanthate 3 was isolated in rather low yields $(\sim 10 \%)$.

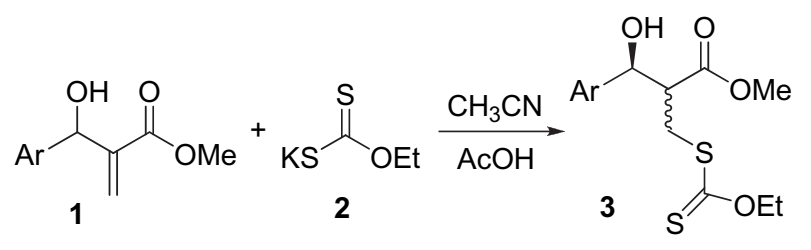

Scheme 1.
Resumen. En este artículo se describe una adición de Michael diastereoselectiva del O-etilditiocarbonato de potasio comercial sobre aductos de Baylis-Hillman, utilizando 9-BBN (9-borabiciclo[3.3.1]no nano) como ácido de Lewis. El 9-BBN protegió el grupo hidroxilo y activó al mismo tiempo el grupo carbonilo del aceptor de Michael.

Keywords: Xantatos; aductos de Baylis-Hillman; 9-BBN, ácido de Lewis.

Looking to improve the process, we realized that the hydroxyl function in the structure of the Baylis-Hillman adduct is in a suitable position to hold a Lewis acid, which would automatically activate the carbonyl function in a six-member structure. Thus, it was hypothesized that a borane, specifically a one-hydride transfer borane such as 9-BBN [6], could be useful not only to activate the Michael acceptor but also to protect the hydroxyl group. Thus the first step in the process might be an acid-base reaction between the hydride and the proton of the hydroxyl group, leading to the formation of the complex 4 (Figure 1).

To confirm this hypothesis, several Baylis-Hillman adducts were prepared and subjected to reaction with potassium $\mathrm{O}$ ethyl dithiocarbonate ( 2 equiv), 9-BBN (1.5 equiv), and acetic acid (1 equiv), in acetonitrile at room temperature. Under these conditions, the desired xanthate was obtained generally in good yields (Table 1). Interestingly, we found that the reaction proceeded with some degree of diastereoselectivity, with the anti isomer being the major product at least in the case of $\mathbf{4 b}$. The relative configuration of this latter product was confirmed by X-ray crystallography of the t-butyldimethylsilyl derivative of the major product of $\mathbf{4 b}$ (Figure 2) [7].

This stereochemical outcome might be rationalized in terms of the two possible intermediates depicted in scheme 2 . If we

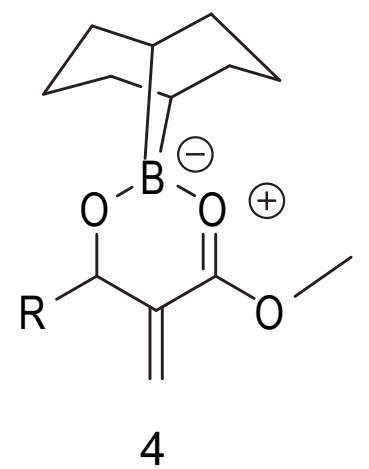

Fig. 1. 
Table 1. Addition of potassium $O$-ethyl dithiocarbonate $O$-ethylxanthate to Baylis-Hillman adducts.

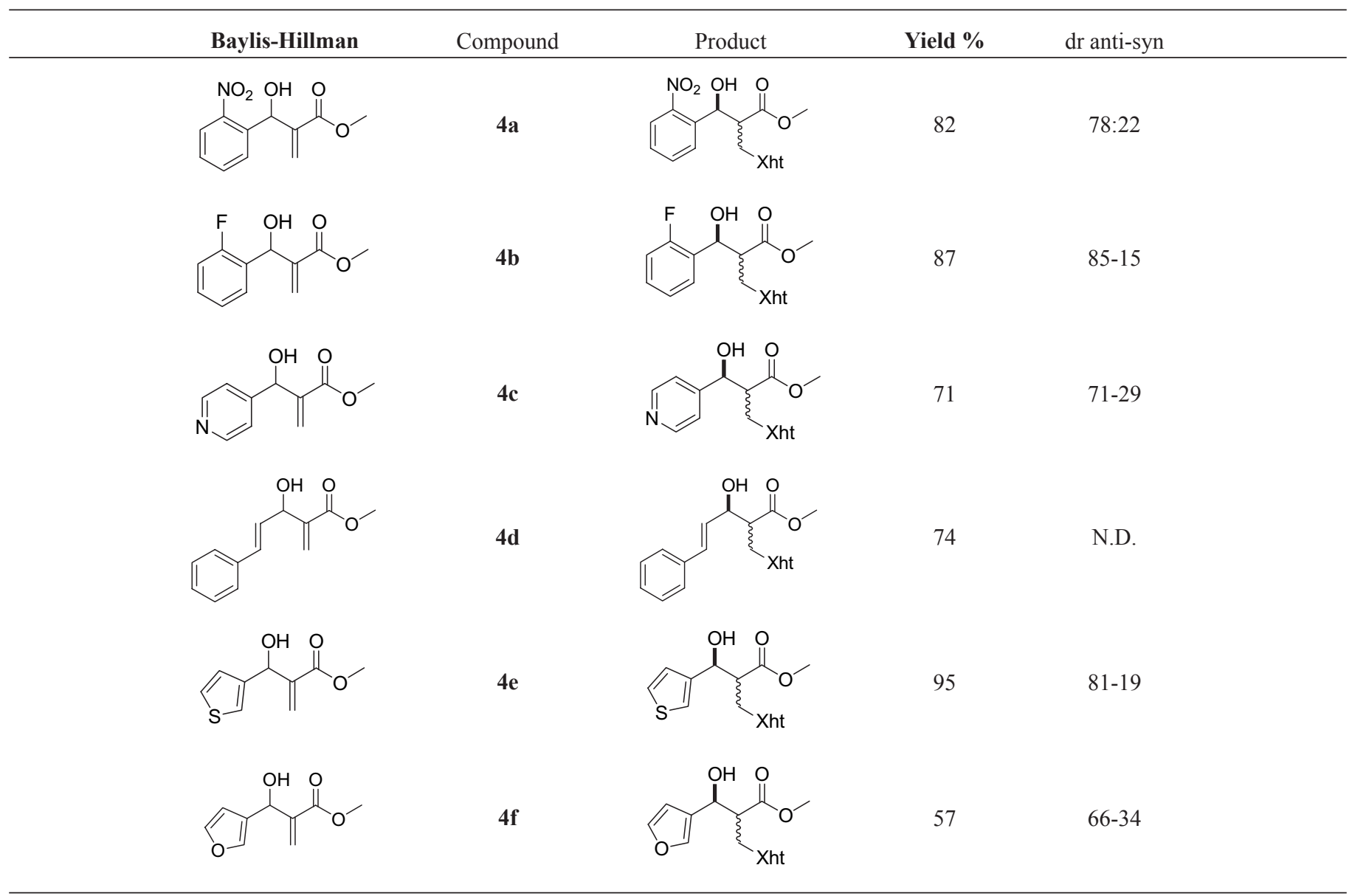

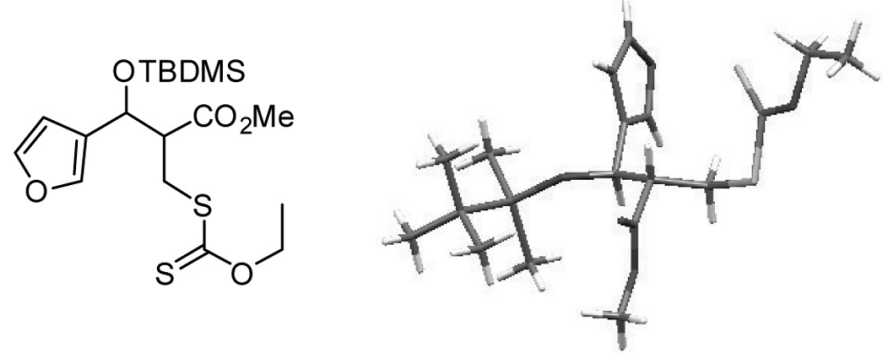

Fig. 2. X-Ray crystallography of the TBDMS-protected major isomer of $4 \mathrm{~b}$.

supposed that the key intermediate generates a six-membered ring, the preferred chair configuration provides the larger functional groups in equatorial position $\mathbf{A}$, avoiding the axial interactions present in the $\mathbf{B}$ intermediate in which the bulky methylenxanthate group is in an axial position. The former enolate A leads to the incoming protonation in the axial position anti with respect to the adjacent hydrogen in the ring. Thus, 9-BBN can play an indispensable role in the stereocontrol of the addition reaction.

\section{Conclusion}

In closing, an efficient new methodology for synthesizing xanthates via 1,4-addition of xanthates to Baylis-Hillman adducts, promoted by $9-\mathrm{BBN}$ as a Lewis acid with control of the diastereoselectivity, is reported. 9-BBN both protects the hydroxyl group of the Baylis-Hillman adduct and activates the carbonyl group of the Michael acceptor.

\section{Experimental Section}

For all reactions, a $\sim 0.5 \mathrm{M}$ 9-BBN (Aldrich) solution in tetrahydrofuran was used. All column chromatography purifications were carried out using silica gel (230-400 mesh ASTM; Merck). TLC was performed on precoated aluminum-backed plates with silica gel $60 \mathrm{~F}_{254}(2 \mathrm{~mm}$; Merck), which were developed using standard visualizing agents such as vanillin and a UV fluorescence Spectroline (254 nm) lamp. Melting points were obtained in a Fisher-Jones apparatus and are uncorrected $\left({ }^{\circ} \mathrm{C}\right)$. NMR spectra were recorded in: $(200 \mathrm{MHz})$ 

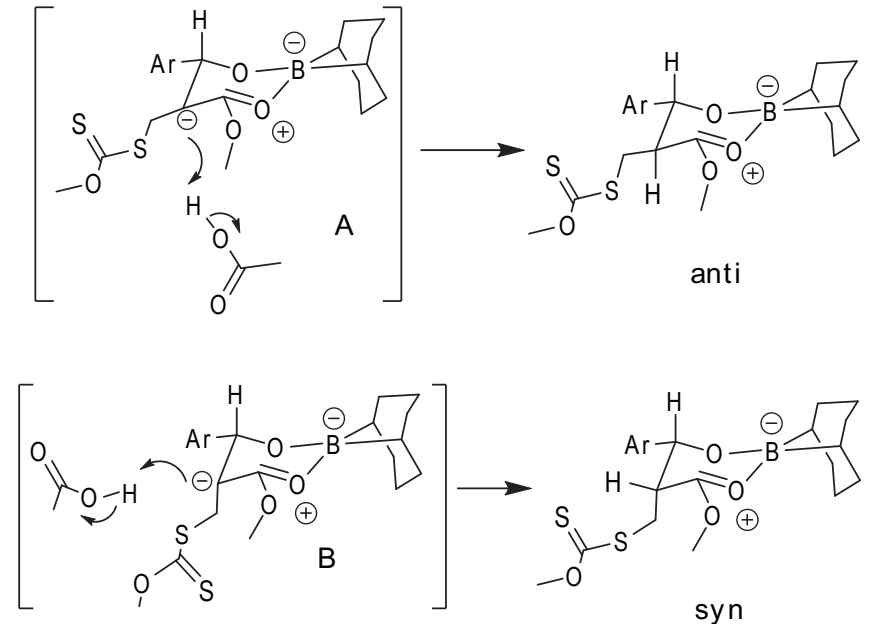

Scheme 2.

Varian Gemini FT 200A, (300 MHz) Eclipse JEOL, (300 MHz) UNITY 300; TMS was used as an internal standard and $\mathrm{CDCl}_{3}$ as solvent. The IR spectra were recorded in a Nicolet FT-IR Magna 750 apparatus. MS spectra were obtained in a JEOL JEM-AX505HA (IE, $70 \mathrm{eV}$ ) apparatus; only the molecular ions $\left(\mathrm{M}^{+}\right)$and major peak are reported (as percentages of the base peak).

Baylis-Hillman Reaction (General Conditions) [7].A solution of substrate aldehyde $(1 \mathrm{mmol})$ and methyl acrylate (3 $\mathrm{mmol})$ in $10 \mathrm{~mL}$ of 1,4 -dioxane/water $(1: 1, \mathrm{v} / \mathrm{v})$ was stirred at room temperature in the presence of $100 \mathrm{~mol} \% \mathrm{DABCO}$, and the reaction progress was monitored by TLC. Upon completion, the reaction mixture was partitioned with ethyl acetate $(150 \mathrm{~mL})$ and water $(80 \mathrm{~mL})$. The organic phase was washed with brine $(2 \times 50 \mathrm{~mL})$, dried over anhydrous $\mathrm{Na}_{2} \mathrm{SO}_{4}$, filtered, and concentrated under reduced pressure. The crude product was purified by flash column chromatography on silica gel, using ethyl acetate and hexane as the eluting solvents to give the desired product.

Compounds 4a [8], 4b [9], 4c [8], 4d [10], and 4f [11] are known compounds, and their spectroscopic data matched those reported in the literature. 4e. Yellow oil, rf: 0.25 (hexane AcOEt, 8:2); ${ }^{1} \mathrm{H}$ NMR (200 MHz, $\left.\mathrm{CDCl}_{3}\right)$ \&: 3.11 (s, 1H), 3.74 $(\mathrm{s}, 3 \mathrm{H}), 5.62(\mathrm{~s}, 1 \mathrm{H}), 5.84(\mathrm{dd}, J=1.2,1.2 \mathrm{~Hz}, 1 \mathrm{H}), 6.31$ (dd, $J=0.6,1.0 \mathrm{~Hz}, 1 \mathrm{H}), 7.02$ (dd, $J=4.8,1.4 \mathrm{~Hz}, 1 \mathrm{H}), 7.20-7.30$ $(\mathrm{m}, 2 \mathrm{H}) ;{ }^{13} \mathrm{C} \mathrm{NMR}\left(75 \mathrm{MHz}, \mathrm{CDCl}_{3}\right) \delta: 51.95,69.88,121.82$, 125.95, 126.17, 141.62, 142.91, 166.79; IR $\left(\mathrm{cm}^{-1}\right) 1718(\mathrm{C}=\mathrm{O})$; 3451, (O-H); ME (IE, m/z) (\%) (M) 198 (44), 85 (100).

\section{Xanthate addition (General Procedure)}

A mixture of the Baylis-Hillman adduct $(1 \mathrm{mmol})$ and $0.5 \mathrm{M}$ 9-BBN solution in THF $(1.5 \mathrm{mmol})$ was stirred for $10 \mathrm{~min}$ at room temperature (until hydrogen production ceased), under a nitrogen atmosphere. The THF was then evaporated and the resulting mixture was stirred for 48 hours with the potassium
O-ethyl dithiocarbonate $(2 \mathrm{mmol})$ and acetic acid $(1 \mathrm{mmol})$ in dry acetonitrile $(200 \mathrm{ml})$. When the reaction was completed (as determined by TLC monitoring), the acetonitrile was evaporated, poured into $\mathrm{H}_{2} \mathrm{O}$, and extracted with EtOAc $(3 \times 100 \mathrm{ml})$. The combined organic layers were washed with $\mathrm{H}_{2} \mathrm{O}$ and brine, dried (anhydrous $\mathrm{Na}_{2} \mathrm{SO}_{4}$ ), and evaporated under vacuum. The crude product was purified by column chromatography (silica gel). Two mmol of 9-BBN was added to the pyridine substrate.

Xanthate 4a: Eluted with 7:3 hexane-ethyl acetate. Rf: 0.36; orange oil; ${ }^{1} \mathrm{H}$ NMR $\left(200 \mathrm{MHz}, \mathrm{CDCl}_{3}\right) \delta: 1.41(\mathrm{t}, 3 \mathrm{H}, J=7.2$ $\mathrm{Hz}), 2.69$ (bs, 1H), 3.38-3.52 (m, 3H), 3.58 (s, 3H), 4.65 (q, $2 \mathrm{H}, J=7.2 \mathrm{~Hz}), 5.63(\mathrm{~d}, 1 \mathrm{H}, J=3.8 \mathrm{~Hz}), 7.43-7.51(\mathrm{~m}, 1 \mathrm{H})$, 7.61-7.74 (m, 2H), $8.00(\mathrm{dd}, 1 \mathrm{H}, J=8.1,1.3 \mathrm{~Hz}) ;{ }^{13} \mathrm{C} \mathrm{NMR}$ $\left(75 \mathrm{MHz}, \mathrm{CDCl}_{3}\right) \delta: 13.60,32.04,49.78,52.40,69.59,70.06$, $125.11,128.85,129.23,136.20,147.56,173.41,214.17$; IR (film) 1050, 1221, 1736, $3497 \mathrm{~cm}^{-1}$; MS (EI) $\mathrm{m} / z 360$ (3) [M $\mathrm{M}^{+}$, 29 (100), 77 (83), 220 (69), 188 (66), 104 (64) 55 (57). HRMS (FAB+) calcd for $\mathrm{C}_{14} \mathrm{H}_{18} \mathrm{O}_{6} \mathrm{~N}_{1} \mathrm{~S}_{2} 360.0576$, found 360.0574 .

4a. Minor product from the mixture with the major product- ${ }^{1} \mathrm{H}$ NMR $\left(200 \mathrm{MHz}, \mathrm{CDCl}_{3}\right) \delta: 1.41(\mathrm{t}, 3 \mathrm{H}, J=7.2 \mathrm{~Hz})$, 2.69 (bs, 1H), 3.38-3.52 (m, 3H), 3.58 (s, 3H), 4.65 (q, 2H, $J=$ $7.2 \mathrm{~Hz}), 5.86(\mathrm{~d}, 1 \mathrm{H}, J=3.8 \mathrm{~Hz}), 7.40-7.49(\mathrm{~m}, 1 \mathrm{H}), 7.60-7.76$ $(\mathrm{m}, 2 \mathrm{H}), 8.06(\mathrm{dd}, 1 \mathrm{H}, J=8.1,1.3 \mathrm{~Hz})$.

Xanthate 4b: Eluted with 7:3 hexane-ethyl acetate Rf: 0.49; yellow solid; mp. $44-46{ }^{\circ} \mathrm{C} ;{ }^{1} \mathrm{H}$ NMR $\left(300 \mathrm{MHz} \mathrm{CDCl}_{3}\right) \delta$ : $1.39(\mathrm{t}, 3 \mathrm{H}, J=7.1 \mathrm{~Hz}), 3.25-3.42(\mathrm{~m}, 3 \mathrm{H}), 3.64(\mathrm{~s}, 3 \mathrm{H})$, $4.60(\mathrm{q}, 2 \mathrm{H}, J=7.2 \mathrm{~Hz}), 5.25(\mathrm{~s}, 1 \mathrm{H}), 7.00-7.07(\mathrm{~m}, 1 \mathrm{H})$, 7.14-7.19 (m, 1H), 7.25-7.33 (m, 1H), 7.40-7.45 (m, 1H); ${ }^{13} \mathrm{C}$ NMR (75 MHz, $\left.\mathrm{CDCl}_{3}\right) \delta: 13.60,34.29,50.87,52.11,68.87$, $70.22,115.21,115.50,124.35,127.58,127.92,128.10,129.59$, 129.70, 158.07, 161.33, 173.21, 213.43; IR (film) 1050, 1225, 1735, $3476 \mathrm{~cm}^{-1}$; MS (EI) $\mathrm{m} / z 333$ (3) $\left[\mathrm{M}^{+}\right], 87$ (100), 210 (97), 193 (50), 125 (49), 315 (35). HRMS (FAB+) calcd for $\mathrm{C}_{14} \mathrm{H}_{18} \mathrm{O}_{4} \mathrm{~F}_{1} \mathrm{~S}_{2} 333.0631$, found 333.0630.

4b. Minor product from the mixture with the major product- ${ }^{1} \mathrm{H}$ NMR (300 MHz, $\left.\mathrm{CDCl}_{3}\right) \delta: 1.31$ (t, 3H, $\left.J=7.1 \mathrm{~Hz}\right)$, 3.25-3.42 (m, 3H), 3.71 (s, 3H), 4.60 (q, 2H, $J=7.2 \mathrm{~Hz}), 5.43$ $(\mathrm{s}, 1 \mathrm{H}), 7.01-7.08(\mathrm{~m}, 1 \mathrm{H}), 7.14-7.21(\mathrm{~m}, 1 \mathrm{H}), 7.26-7.34(\mathrm{~m}$, 1H), 7.49-7.55 (m, 1H).

Xanthate 4c: Eluted with 96:4 ethyl acetate-methanol Rf: 0.39; yellow oil; ${ }^{1} \mathrm{H}$ NMR $\left(300 \mathrm{MHz}, \mathrm{CDCl}_{3}\right) \delta: 1.41(\mathrm{t}, 3 \mathrm{H}, J$ $=7.1 \mathrm{~Hz}), 3.24-3.50(\mathrm{~m}, 3 \mathrm{H}), 3.62(\mathrm{~s}, 3 \mathrm{H}), 4.63(\mathrm{q}, 2 \mathrm{H}, J=7.2$ $\mathrm{Hz}), 5.02$ (d, 1H, $J=4.8 \mathrm{~Hz}), 7.34$ (d, 2H, $J=5.7 \mathrm{~Hz}), 8.52-$ $8.54(\mathrm{~m}, 2 \mathrm{H}) ;{ }^{13} \mathrm{C}$ NMR $\left(75 \mathrm{MHz}, \mathrm{CDCl}_{3}\right) \delta: 13.66,34.48$, 51.17, 52.11, 70.43, 70.60, 121.36, 148.75, 151.83, 172.48, 213.78; IR (film) 1050, 1222, 1737; $3224 \mathrm{~cm}^{-1}$; MS (EI) $\mathrm{m} / z$ 316 (2) $\left[\mathrm{M}^{+}\right], 108$ (100), 89 (53), 57 (47), 180 (17). HRMS (FAB+) calcd for $\mathrm{C}_{13} \mathrm{H}_{18} \mathrm{O}_{4} \mathrm{~N}_{1} \mathrm{~S}_{2} 316.0677$, found 316.0670 .

4c. Minor product from the mixture with the major product- ${ }^{1} \mathrm{H}$ NMR (300 MHz, $\left.\mathrm{CDCl}_{3}\right) \delta: 1.33(\mathrm{t}, 3 \mathrm{H}, J=7.1 \mathrm{~Hz}), 3.12-3.45$ (m, 3H), 3.67 (s, 3H), 4.55 (q, 2H, J=7.2 Hz), 5.19 (d, 1H, $J$ $=4.8 \mathrm{~Hz}), 7.40(\mathrm{~d}, 2 \mathrm{H}, J=5.7 \mathrm{~Hz}), 8.52-8.54(\mathrm{~m}, 2 \mathrm{H})$. 
Xanthate 4d: Eluted with 7:3 hexane-ethyl acetate Rf: 0.42; yellow solid; mp. $74-75{ }^{\circ} \mathrm{C} ;{ }^{1} \mathrm{H}$ NMR $\left(300 \mathrm{MHz} \mathrm{CDCl}_{3}\right) \delta$ : $1.40(\mathrm{t}, 3 \mathrm{H}, J=7.2 \mathrm{~Hz}), 2.86(\mathrm{bs}, 1 \mathrm{H}), 3.07-3.13(\mathrm{dd}, 1 \mathrm{H}$, $J=5.4,5.7 \mathrm{~Hz}), 3.34-3.56(\mathrm{~m}, 2 \mathrm{H}), 3.72(\mathrm{~s}, 3 \mathrm{H}),, 4.62(\mathrm{q}$, $2 \mathrm{H}, J=7.2 \mathrm{~Hz}), 4.55(\mathrm{~m}, 1 \mathrm{H}), 6.23(\mathrm{dd}, 1 \mathrm{H}, J=15.8,6.2$ $\mathrm{Hz}), 6.67$ (dd, $1 \mathrm{H}, J=15.8,1.2 \mathrm{~Hz}), 7.24-7.39(\mathrm{~m}, 5 \mathrm{H})$; ${ }^{13} \mathrm{C} \mathrm{NMR}\left(75 \mathrm{MHz}, \mathrm{CDCl}_{3}\right) \delta: 13.64,29.62,50.82,51.96$, $70.12,72.96,126.65,127.94,128.56,128.79,132.30,136.38$, 172.91, 213.96; IR (film) 1049, 1222, 1734, $3455 \mathrm{~cm}^{-1}$; MS (EI) $m / z 340$ (2) $\left[\mathrm{M}^{+}\right], 218$ (100), 133 (69), 87 (52), 131 (45) 55 (42). HRMS (FAB+) calcd for $\mathrm{C}_{16} \mathrm{H}_{20} \mathrm{O}_{4} \mathrm{~S}_{2} 340.0803$, found 340.0807 .

4d. Minor product from the mixture with the major product- ${ }^{1} \mathrm{H}$ NMR $\left(300 \mathrm{MHz}, \mathrm{CDCl}_{3}\right) \delta: 1.36(\mathrm{t}, 3 \mathrm{H}, J=7.2 \mathrm{~Hz})$, 2.86 (bs, 1H), 3.07-3.13 (dd, $1 \mathrm{H}, J=5.4,5.7 \mathrm{~Hz}), 3.23-3.44$ (m, 2H), 3.70 (s, 3H,), 4.62 (q, 2H, $J=7.2 \mathrm{~Hz}), 5.18(\mathrm{~m}, 1 \mathrm{H})$, $6.25(\mathrm{dd}, 1 \mathrm{H}, J=15.8,6.2 \mathrm{~Hz}), 6.62(\mathrm{dd}, 1 \mathrm{H}, J=15.8,1.2$ $\mathrm{Hz}), 7.24-7.39$ (m, 5H).

Xanthate 4e: Eluted with 7:3 hexane-ethyl acetate Rf: 0.5; yellow solid; mp. $44-46{ }^{\circ} \mathrm{C} ;{ }^{1} \mathrm{H}$ NMR $\left(200 \mathrm{MHz} \mathrm{CDCl}_{3}\right) \delta$ : $1.40(\mathrm{t}, 3 \mathrm{H}, J=7.1 \mathrm{~Hz}), 2.63(\mathrm{bs}, 1 \mathrm{H}), 3.24-3.44(\mathrm{~m}, 3 \mathrm{H})$, 3.69 (s, 3H), 4.63 (q, 2H, $J=7.2 \mathrm{~Hz}), 5.04$ (d,1H, $J=5.4 \mathrm{~Hz}$ ), 7.07 (dd, $1 \mathrm{H}, J=4.8,1.4 \mathrm{~Hz}), 7.24-7.36(\mathrm{~m}, 2 \mathrm{H}) ;{ }^{13} \mathrm{C} \mathrm{NMR}$ $\left(50 \mathrm{MHz}, \mathrm{CDCl}_{3}\right) \delta: 13.69,34.40,51.18,52.19,70.26,70.95$, $121.97,125.51,126.58,142.45,173.42,213.78$; IR (film) 1048, 1221, 1733, $3471 \mathrm{~cm}^{-1}$; MS (EI) $m / z 319$ (4) [MH'], 198 (100), 181 (92), 85 (70), 302 (60). HRMS (FAB+) calcd for $\mathrm{C}_{12} \mathrm{H}_{16} \mathrm{O}_{4} \mathrm{~S}_{3} 320.0211$, found 320.0208 .

4e. Minor product from the mixture with the major product- ${ }^{1} \mathrm{H}$ NMR $\left(200 \mathrm{MHz}, \mathrm{CDCl}_{3}\right) \delta: 1.39$ (t, 3H, $\left.J=7.1 \mathrm{~Hz}\right)$, 2.63 (bs, 1H), 3.20-3.48 (m, 3H), 3.69 (s, 3H), 4.58 (q, 2H, $=7.2 \mathrm{~Hz}), 5.20(\mathrm{~d}, 1 \mathrm{H}, J=5.4 \mathrm{~Hz}), 7.07(\mathrm{dd}, 1 \mathrm{H}, J=4.8,1.4$ $\mathrm{Hz}), 7.24-7.36$ (m, 2H).

Xanthate 4f: Eluted with 7:3 hexane-ethyl acetate Rf: 0.43 ; yellow oil; ${ }^{1} \mathrm{H}$ NMR $\left(300 \mathrm{MHz}, \mathrm{CDCl}_{3}\right) \delta: 1.41(\mathrm{t}, 3 \mathrm{H}, J=7.2$ $\mathrm{Hz}), 2.17$ (s, 1H), 3.18-3.50 (m, 3H), 3.72 (s, 3H), 4.64 (q, 2H, $J=7.2 \mathrm{~Hz}), 4.94$ (d, 1H, $J=5.4 \mathrm{~Hz}), 6.39-6.40(\mathrm{~m}, 1 \mathrm{H}), 7.41-$ $7.43(\mathrm{~m}, 2 \mathrm{H}) ;{ }^{13} \mathrm{C} \mathrm{NMR}\left(75 \mathrm{MHz}, \mathrm{CDCl}_{3}\right) \delta: 13.73,34.37$, 50.54, 52.22, 67.52, 70.31, 108.42, 126.08, 139.74, 143.68, 173.42, 213.77; IR (film) 1051, 1220, 1738, $3454 \mathrm{~cm}^{-1}$; MS (EI) $m / z 318$ (2) $\left[\mathrm{M}^{+}\right], 182$ (100), 87 (29), 97 (15).

4f. Minor product from the mixture with the major product- ${ }^{1} \mathrm{H}$ NMR $\left(300 \mathrm{MHz}, \mathrm{CDCl}_{3}\right) \delta: 1.38(\mathrm{t}, 3 \mathrm{H}, J=7.2 \mathrm{~Hz})$, 2.17 (s, 1H), 3.18-3.50 (m, 3H), 3.70 (s, 3H), 4.60 (q, 2H, $J=$ $7.2 \mathrm{~Hz}), 5.53(\mathrm{~d}, 1 \mathrm{H}, J=5.4 \mathrm{~Hz}), 6.39-6.40(\mathrm{~m}, 1 \mathrm{H}), 7.38-7.40$ $(\mathrm{m}, 2 \mathrm{H})$.

\section{6e (Alcohol protection)}

A mixture of the xanthate $(1 \mathrm{mmol})$, imidazol $(2 \mathrm{mmol})$, and $\mathrm{TBSCl}(1.1 \mathrm{mmol})$ in DMF $(2 \mathrm{ml} / \mathrm{g}$ de MP), was stirred at room temperature under a nitrogen atmosphere for 5 days. When the reaction was completed (monitored by TLC), the mixture was poured into $\mathrm{H}_{2} \mathrm{O}(10 \mathrm{ml})$, and extracted with EtOAc $(3 \times 5 \mathrm{ml})$. The combined organic layers were washed with $\mathrm{H}_{2} \mathrm{O}$, brine, dried (anhyd $\mathrm{Na}_{2} \mathrm{SO}_{4}$ ), and evaporated. The crude product was purified by column chromatography (silica gel). The colorless product was obtained by slow precipitation from methanol/ hexane. Eluted with 8:2 hexane-ethyl acetate Rf: 0.55; white solid; mp. 53-54 ${ }^{\circ} \mathrm{C}$; ${ }^{1} \mathrm{H}$ NMR $\left(200 \mathrm{MHz} \mathrm{CDCl}_{3}\right) \delta: 0.24(\mathrm{~s}$, $3 \mathrm{H}),-0.01(\mathrm{~s}, 3 \mathrm{H}), 0.80(\mathrm{~s}, 9 \mathrm{H}), 1.33(\mathrm{t}, 3 \mathrm{H}, J=7.2 \mathrm{~Hz}), 2.91-$ $3.21(\mathrm{~m}, 3 \mathrm{H}), 3.69(\mathrm{~s}, 3 \mathrm{H}), 4.55(\mathrm{q}, 2 \mathrm{H}, J=7.1 \mathrm{~Hz}), 4.95(\mathrm{~d}$, $1 \mathrm{H}, J=7.8 \mathrm{~Hz}), 7.03-7.06(\mathrm{dd}, 1 \mathrm{H}, J=5.1,1.3 \mathrm{~Hz}), 7.13-7.16$ $(\mathrm{m}, 1 \mathrm{H}), 7.27-7.31(\mathrm{~m}, 1 \mathrm{H}) ;{ }^{13} \mathrm{C} \mathrm{NMR}\left(50 \mathrm{MHz}, \mathrm{CDCl}_{3}\right) \delta=$ $-5.40,-4.91,13.72,17.98,25.56,33.86,51.88,53.58,70.01$, $72.35,122.29,126.01,126.15,142.64,172.83,213.59$; IR (film) 839, 1051, 1085, 1221, 1739, $\mathrm{C}=\mathrm{O} \mathrm{cm}$ cm $^{-1} \mathrm{MS}$ (EI) $\mathrm{m} / \mathrm{z}$ $434(3)\left[\mathrm{MH}^{+}\right], 255(100)$.

\section{Acknowledgements}

Financial support from the DGAPA-UNAM (project PAPIITIN213407) is gratefully acknowledged and Dr. Joseph M. Muchowski for many helpful discussions. Also we thank $\mathrm{H}$. Rios, R. Patiño, J. Pérez, L. Velasco, N. Zavala, E. Hernández, M. I. Chávez and A. Peña for technical support and R. A. Toscano and S. Hernández for X-ray crystallography.

\section{References}

1. Basavaiah, D.; Rao, A.; Satyanarayana, T. Chem. Rev. 2003, 103, 811-891.

2. a) Pringle, W.; Sharpless, B.. Tetrahedron Lett. 1999, 40, 51515154. b) Shi, M.; Jiang, J.; Cui, S.; Feng, Y.. J. Chem. Soc., Perkin Trans 1. 2001, 390-393.

3. a) Basavaiah, D.; Dhrama Rao, P.; Suguna Hyma, R. Tetrahedron. 1996, 52, 8001-8062. b) Bulliard, M.; Zeitz, H.; Giese, B. Synlett 1991, 423-424. c) Kamimura, A.; Morita, R.; Matsuura, K.; Omata, Y.; Shirai, M. Tetrahedron Lett. 2002, 43, 6189-6191.

4. a) Zard, S.Z. Angew. Chem. Int. Ed. Engl. 1997, 36, 672-685. b) Quiclet-Sire, B.; Zard, S.Z. Chem. Eur. J. 2006, 12, 6002-6016.

5. Bernard, J.; Favier, A.; Zhang, L.; Nilasaroya, A.; Davis, T.P.; Barner-Kowollik, C.; Stenzel, M.H. Macromolecules. 2005, 38, 5475-5484.

6. a) Stentiford, R. Synlett. 2000, 1, 159. b) Bar-Haim, G.; Kol, M. Org. Lett 2004, 6, 3549-3551. c) Syed, B.M.; Gustafsson, T.; Kihlberg, J. Tetrahedron. 2004, 60, 5571-5575. d) Gawdzik, B.; Iwanek, W. Tetrahedron Assymm. 2005, 16, 2019-2023.

7. Crystal structure has been deposited at the Cambridge Crystallographic Data Centre: CCDC (739264). Unit cell parameters: a $6.6820(7)$ b 7.3796(8) c 23.075(3), alpha 87.113(2) beta 81.463(2) gamma 83.056(2), space group P-1.

8. Mi, X.; Luo, S.; Cheng, J.-P. J. Org. Chem. 2005, 70, 2338-2341.

9. Coelho, F.; Almeida, W. P.; Veronese, D.; Mateus, C. R.; Silva Lopes, E. C.; Rossi, R. C.; Silveira, G. P. C.; Pavam, C. H Tetrahedron 2002, 58, 7437-7447.

10. (a) Sa, M. M.; Ramos, M. D.; Fernandes, L. Tetrahedron 2006, 62, 11652-11656. (b) Ciganek, E. Organic Reactions (Hoboken, NJ, United States) 1997, pp. 51.

11. Patil, S. N.; Liu, F. J. Org. Chem. 2007, 72, 6305-6308. 